\title{
Initiator-Integrated 3D Printing Enables the Formation of Complex Metallic Architectures
}

\author{
Xiaolong Wang, ${ }^{\dagger, \ddagger, \|}$ Qiuquan Guo, ${ }^{\ddagger}, \|$ Xiaobing Cai, ${ }^{\ddagger}$ Shaolin Zhou, ${ }^{\ddagger}$ Brad Kobe, ${ }^{\S}$ and Jun Yang ${ }^{*}$, \\ ${ }^{\dagger}$ State Key Laboratory of Solid Lubrication, Lanzhou Institute of Chemical Physics, Chinese Academy of Sciences, Lanzhou 730000, \\ China \\ ${ }^{\ddagger}$ Department of Mechanical and Materials Engineering, The University of Western Ontario, London, Ontario N6A 5B9, Canada \\ ${ }^{\S}$ Surface Science Western, The University of Western Ontario, London, Ontario N6G 0J3, Canada
}

\section{Supporting Information}

ABSTRACT: Three-dimensional printing was used to fabricate various metallic structures by directly integrating a Br-containing vinyl-terminated initiator into the $3 \mathrm{D}$ resin followed by surface-initiated atomic-transfer radical polymerization (ATRP) and subsequent electroless plating. $\mathrm{Cu}$ - and Ni-coated complex structures, such as microlattices, hollow balls, and even Eiffel towers, were prepared. Moreover, the method is also capable of fabricating ultralight cellular metals with desired structures by simply etching the polymer template away. By combining the merits of $3 \mathrm{D}$ printing in structure design with those of ATRP in surface modification and polymer-assisted ELP of metals, this universal, robust, and cost-effective approach has largely extended the capability of $3 \mathrm{D}$ printing and will make $3 \mathrm{D}$ printing technology more practical in areas of electronics, acoustic absorption, thermal insulation, catalyst supports, and others.

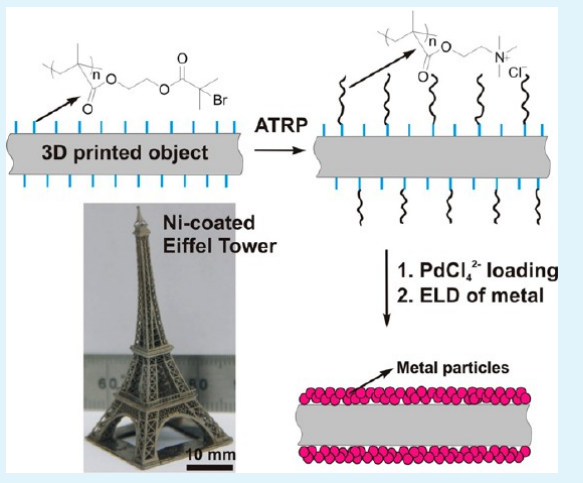

KEYWORDS: 3D printing, metallization, electroless deposition, atomic-transfer radical polymerization

\section{INTRODUCTION}

Three-dimensional (3D) printing, an enabling fabrication technology, allows one to create sophisticated, custome-made, low-cost devices that were either impossible to produce or required complicated procedures and manufacturing facilities. ${ }^{1}$ With the capability of rapidly turning materials into functional devices, this revolutionary technology is increasingly attracting attention from various fields such as engineering, biology, chemistry, and materials science. However, in spite of its utilization in tissue and scaffold engineering ${ }^{2-8}$ and industrial rapid prototyping, 9,10 only a handful of other applications have been addressed to date, such as specialized microfluidics ${ }^{11}$ and electronics. ${ }^{12,13}$ It is therefore important to develop and extend $3 \mathrm{D}$ printing capabilites to meet the specific requirements for various applications; however, developing specific printing strategies together with many different material/ink systems is quite costly and time-consuming. Another approach is to produce functionalized materials/devices by simple modification of the present $3 \mathrm{D}$ printing technologies. ${ }^{14-16}$ Among these, integrating active components into $3 \mathrm{D}$ printing has proven to be a successful method. ${ }^{17,18}$ Recently, 3D reactionware for chemical synthesis and analysis were printed on a low-cost Fab@Home robocasting platform by integrating conductive carbon black and catalytic $\mathrm{Pd} / \mathrm{C}$. This could potentially be applied to customized chemistry and healthcare wares by only a few mouse clicks in the near future. ${ }^{18}$ It is evident that the integrated strategy is capable of extending 3D printing to various applications by simply selecting different additives. As a matter of fact, our very recently developed initiator-integrated 3D printing approach, 3 DP, ${ }^{19,20}$ has opened a wider window for $3 \mathrm{D}$ printing in practical applications in biomedical science, microfluidics, composites, and electronics, where surface properties are critical.

Herein, we demonstrate the use of the i3DP strategy to make metallic structures/materials that have complex geometries. Over the last few years, metallic structures, especially ultralight cellular metals, have attracted a great deal of interest because of their superior electrical, mechanical, and thermal properties. ${ }^{15}$ To advance this emerging field, two major strategies have been suggested, namely, additive printing of metal alloys or metallic oxides and polymer-template-directed metallization. In the former, low-melting-point metal alloys or nanoparticle suspensions were printed layer-by-layer to form 3D structures. $^{13,21-24}$ However, this method is only compatible with specific alloys or suspensions. Futhermore, corresponding nanoparticles and suspensions must be developed for each metal structure, which is costly and time-consuming. In the latter, polymer templates were first fabricated using stereolithography, on which the subsequent electroless plating (ELP) of metals was employed to realize metallic structures. For example, ultralight $\mathrm{Ni}$ microlattices have been achieved using this strategy. ${ }^{25-27}$ However, apart from producing ordered and

Received: November 13, 2013

Accepted: December 11, 2013

Published: December 11, 2013 
hierarchical structures, the sterolithography used here, which is mostly based on photopolymer waveguide prototyping, is not suitable to produce structures with complex geometries. Moreover, for the latter strategy, ELP was carried out only on physically absorped catalysts; thus, the poor realiability, uniformity, and duriability of the resultant metallic sturctures are always concerns because of the weak adhesion of the metal layer to substrate. Accordingly, producing high-quality metallic structures/materials on a large scale still remains a challenge.

We addressed the challenge of $i 3 \mathrm{DP}$ by growing polyelectrolyte brushes via atomic-transfer radical polymerization (ATRP) and subsequent ELP of metals. This method was inspired by the previous findings of iPDMS ${ }^{28-30}$ as well as the fact that polyelectrolyte brushes are capable of being nano-thick platforms to induce metal ELP to form metal layers with excellent adhesion to the substrate. ${ }^{31-35}$ By directly introducing the vinyl-terminated initiator into the photo-curable resin of the $3 \mathrm{D}$ printer, the initiator would then be fully integrated into the highly cross-linked networks of 3D printed architechtures. Then, dense and uniform polyelectrolyte brushes can be grown via surface-initiated ATRP, and, finally, the surface-tethered polyelectrolyte-brushes-assisted ELP results in high-quality metallic microarchitechtures. As a proof of concept, a MiiCraft $3 \mathrm{D}$ printer that creates $3 \mathrm{D}$ objects by photo-polymerizing the acrylate-based liquid resin in a layer-by-layer sequence was used to demonstrate the universal approach for fabricating complex metallic structures. The computer-aided process makes the structure and dimensions of the target architectures digitally adjustable and the automatic printing highly effective. Hence, after the subsequent ATRP of electrolyte monomers and electroless plating, we were able to fabricate various desired metallic objects, such as $\mathrm{Ni}$ - and $\mathrm{Cu}$-coated microlattices, hollow balls, and even metallic Eiffel towers for the first time in a programmable way without any specialized, expensive processes or instruments. Notably, the polymer core of the resultant metallic structures can be etched away to produce ultralight cellular metals with desired structures.

\section{EXPERIMENTAL SECTION}

Materials. Three-dimensional printer-resin base was purchased from MiiCraft. All other chemicals and solvents were obtained from Sigma-Aldrich and used as received unless otherwise indicated. $\mathrm{CuBr}$ was purified by reflux in acetic acid. Initiator 2-(2bromoisobutyryloxy)ethyl methacrylate (BrMA) and monomer 2(methacryloyloxy)ethyl-trimethylammonium chloride (METAC) were passed through an aluminum oxide column to remove inhibitors prior to use.

Preparation of Initiator-Containing Resin. To prepare the initiator-containing resin, BrMA was added to the resin base of the MiiCraft 3D printer and mixed well. After degassing for 30 minutes in the dark, the customized resin containing 5 wt \% initiator BrMA was ready for printing.

Initiator-Integrated Architectures Created by 3D Printing. The MiiCraft 3D printer is based on stereolithography, which creates $3 \mathrm{D}$ objects by photo-polymerizing the acrylate-based liquid resin in a layer-by-layer sequence. A solid $3 \mathrm{D}$ model is designed using a CAD tool and digitally sliced into a series of 2D layers, which are then used to control the photo projection to solidify the liquid resin in a tank. After one layer is solidified at the bottom of the tank, an elevator lifts the printed object up, and a new cylce starts solidifying. The entire process is repeated until the whole $3 \mathrm{D}$ object is formed. For the $5 \mathrm{wt}$ $\%$ initiator-containing customized resin, the exposure time for each layer is 3.5 seconds, and the lift height is $50 \mu \mathrm{m}$. The parameters are the same for the MiiCraft resin base, indicating that the added initiators do not show any negative effect on printing. Once finished, the printed architecture was ultrasonically rinsed for $5 \mathrm{~min}$ using ethanol to remove any physically absorbed resin and small molecular monomers, dried by nitrogen flow, and postcured by UV light for 15 $\min$.

PMETAC Brushes Grown by Surface-Initiated ATRP. The typical process is as follows: $4.6 \mathrm{~g}$ of commercially available METAC was dissolved in $5 \mathrm{~mL}$ of methanol at $20^{\circ} \mathrm{C}$ and degassed for $20 \mathrm{~min}$ by passing a continuous stream of dry $\mathrm{N}_{2}$ through the solution as it is stirred. 2,2-Dipyridyl $(0.24 \mathrm{~g})$ and $\mathrm{CuBr}(0.06 \mathrm{~g})$ were then added to the solution. The mixture was further stirred and degassed with a stream of dry $\mathrm{N}_{2}$ for $15 \mathrm{~min}$. The 3D printed initiator-integrated samples were immersed into the mixture. The polymerizations were then performed at $60{ }^{\circ} \mathrm{C}$ under nitrogen protection for $4 \mathrm{~h}$. After polymerization, the samples were removed, washed with methanol and water, and then dried under a stream of $\mathrm{N}_{2}$.

Electroless Plating (ELP). The PMETAC-coated 3D samples were immersed into a $5 \mathrm{mM}\left(\mathrm{NH}_{4}\right)_{2} \mathrm{PdCl}_{4}$ aqueous solution for 15 min, where $\mathrm{PdCl}_{4}{ }^{2-}$ moieties were immobilized onto the brushes by ion exchange because of their high affinity to quaternary ammonium positive ions. Then, after rinsing thoroughly with water, the $\mathrm{PdCl}_{4}{ }^{2-}$ moieties-loaded samples were immersed into the house-made plating bath to obtain metallic architectures. $\mathrm{Cu}$ - and Ni-coated structures were prepared in this case. The $\mathrm{Cu}$ plating bath contained a 1:1 mixture of freshly prepared solutions $\mathrm{A}$ and $\mathrm{B}$. Solution A consisted of $12 \mathrm{~g} / \mathrm{L}$ of $\mathrm{NaOH}, 13 \mathrm{~g} / \mathrm{L}$ of $\mathrm{CuSO}_{4} \cdot 5 \mathrm{H}_{2} \mathrm{O}$, and $29 \mathrm{~g} / \mathrm{L}$ of potassium sodium tartrate. Solution B was $9.5 \mathrm{~mL} / \mathrm{L}$ of $\mathrm{HCHO}$ in water. The electroless plating of $\mathrm{Ni}$ was performed in the following steps using a plating bath consisting of $40 \mathrm{~g} / \mathrm{L}$ of $\mathrm{Ni}_{2} \mathrm{SO}_{4} \cdot 5 \mathrm{H}_{2} \mathrm{O}, 20 \mathrm{~g} / \mathrm{L}$ of sodium citrate, $10 \mathrm{~g} / \mathrm{L}$ of lactic acid, and $1 \mathrm{~g} / \mathrm{L}$ of dimethylamine borane (DMAB) in water. A nickel stock solution of all components except the $D M A B$ reductant was prepared in advance. $A D M A B$ aqueous solution was prepared separately. The stock solutions were prepared for a 4:1 volumetric proportion of nickel-to-reductant stocks in the final electroless bath.

Template Etching for Cellular Lattice. The $4 \times 4 \times 8 \mathrm{~mm}^{3}$ microlattice was immersed into a $3 \mathrm{M} \mathrm{NaOH}$ aqueous solution and kept at $60{ }^{\circ} \mathrm{C}$ for $24 \mathrm{~h}$. Next, the microlattice was washed carefully using DI water several times. Finally, it was dried overnight in vacuum at $60^{\circ} \mathrm{C}$.

Characterization. Surface chemical-composition information of 3D printed microlattices was obtained by X-ray photoelectron spectroscopy (XPS). The measurements were carried out using a Kratos Axis Ultra spectrometer using a monochromatic $\mathrm{Al} \mathrm{K} \alpha$ radiation source. The binding energies were referenced to the $\mathrm{C} 1 \mathrm{~s}$ line at $284.8 \mathrm{eV}$ from adventitious carbon. Using an argon ion gun (Kratos Minibeam III) to etch the samples, the depth profile of the $\mathrm{Br}$ lattice was measured. The argon ion gun was operated at $4 \mathrm{kV}$ and a 15 $\mathrm{mA}$ emission current. The sputtered area is $3 \mathrm{~mm} \times 3 \mathrm{~mm}$. The sputter rate is $1.3 \mathrm{~nm} / \mathrm{min}$ for $\mathrm{Al}_{2} \mathrm{O}_{3}$, and the etching depth for the polymer is estimated to be about $5 \mathrm{~nm} / \mathrm{min}$. The morphology of the resultant metallic architectures was investigated using a Hitachi S-4500 field-emission scanning electron microscope at a $5 \mathrm{kV}$ accelerating voltage.

\section{RESULTS AND DISCUSSION}

Scheme 1 illustrates the fabrication process. In a typical experiment, a Br-containing vinyl-terminated initiator, 2-(2bromoisobutyryloxy)ethyl methacrylate, was mixed with the original photo-curable resin to form a customized resin with 5 wt $\%$ initiator. After 3D printing, the customized resin formed highly cross-linked networks with the initiator integrated both inside and on the surface of the printed objects (microlattice in Scheme 1, Br-lattice). Subsequently, the outermost $\mathrm{Br}$ groups initiated the ATRP process to grow a layer of PMETAC on the surface of the microlattices (PMETAC-lattice). After that, the samples were immersed in a $\left(\mathrm{NH}_{4}\right)_{2} \mathrm{PdCl}_{4}$ aqueous solution for 15 min to immobilize $\mathrm{PdCl}_{4}{ }^{2-}$ onto the polymer brushes because of their higher affinity to quaternary ammonium groups 
Scheme 1. Schematic Illustration of the Fabrication of Complex Metallic Architectures by Initiator-Integrated 3D Printing Followed by SI-ATRP and Subsequent Electroless Plating $^{a}$
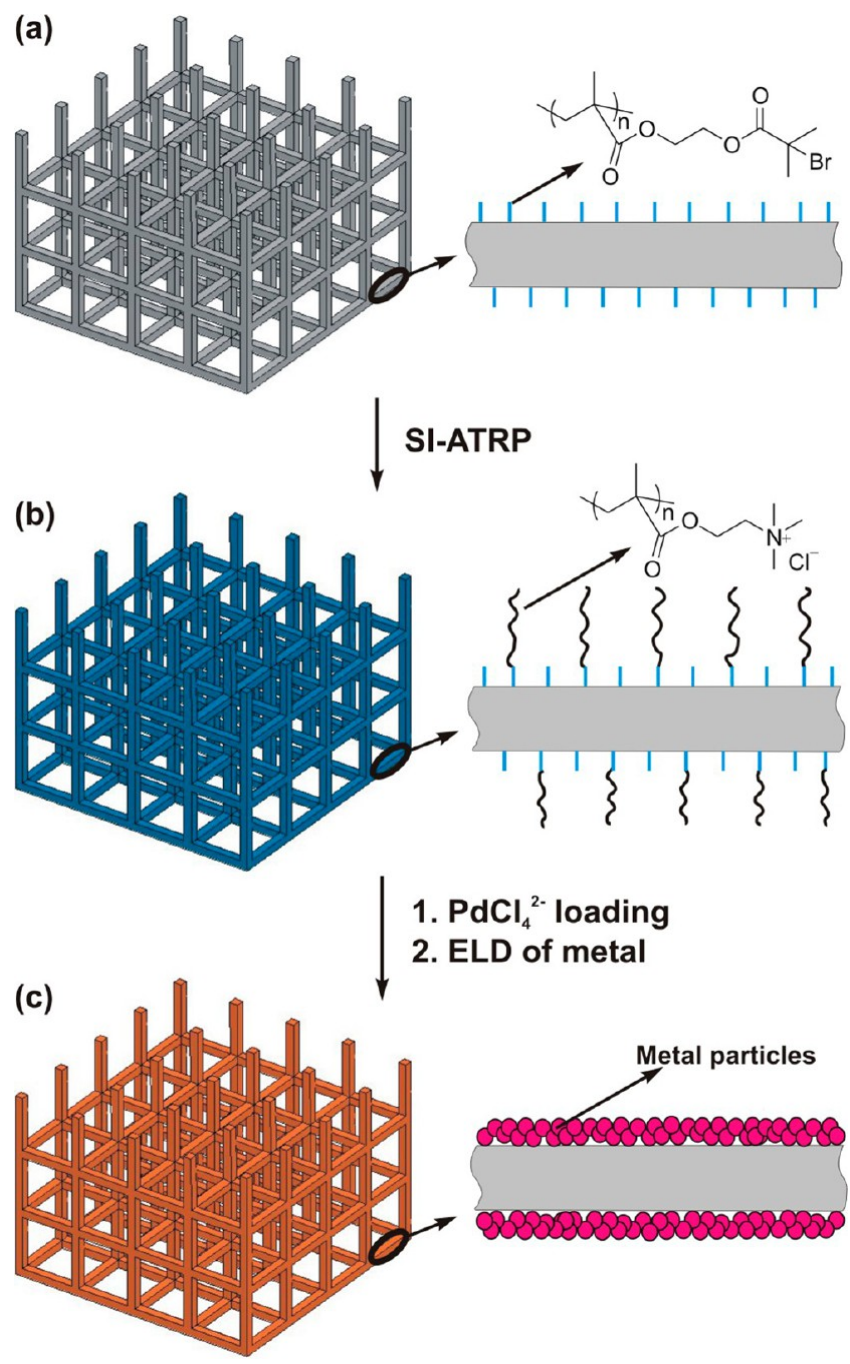

${ }^{a}$ (a) Three-dimensional-printed initiator-integrated microlattice, (b) PMETAC-modified microlattice, and (c) metal-coated microlattice.

(PMETAPd-lattice). Finally, metallic materials with complex geometries were obtained by immersion into a metal ELP bath for $25 \mathrm{~min}$ at room temperature. Copious rinsing was carried out at the end of each step to avoid any physisorption of unattached chemicals.

X-ray photoelectron spectroscopy (XPS) was first carried out to monitor the entire fabrication process. The survey scan for the Br-lattice (Figure 1a) clearly shows the characteristic $\mathrm{Br} 3 \mathrm{~d}$ peak at $68.8 \mathrm{eV}$, which is completely absent for the printed lattice using the original resin base (Figure S1). As observed by the XPS depth profile (Figure $1 \mathrm{~b}$ ), the characteristic signal of $\mathrm{Br}$ existed from the outermost surface to hundreds of nanometers and even dozens of micrometers beneath the surface, which strongly verifies that the initiator has been integrated into the whole $3 \mathrm{D}$ printed architecture as expected. On the surface, $\mathrm{Br}$ is 0.3 atom \%, whereas the XPS depth profile shows that it slightly increased to $0.7 \%$ at a depth of hundreds of nanometers. Interestingly, a sharp increase in $\mathrm{C}$ from $\sim 73.0$ to $\sim 95.0 \%$ and a decrease in $\mathrm{O}$ from 25.0 to $3.5 \%$ after the first
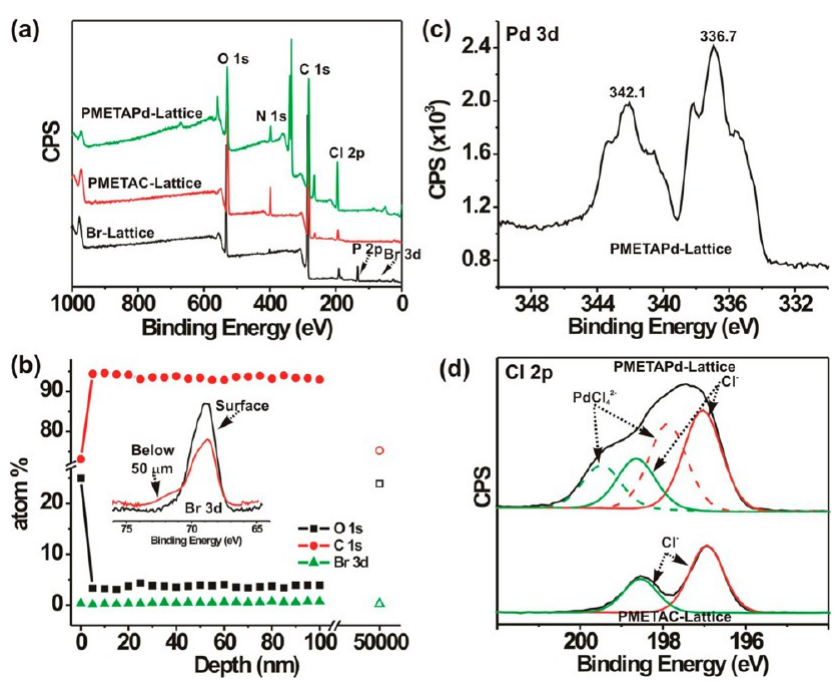

Figure 1. (a) XPS survey spectra of 3D printed initiator-integrated microlattice (Br-lattice) before and after PMETAC grown via SIATRP and subsequent ion exchange (bottom to top). (b) XPS depth profile of Br-lattice. The $50 \mu \mathrm{m}$ depth surface was cut manually, not by etching. The inset is the $\mathrm{Br} 3 \mathrm{~d}$ high-resolution spectra of Br-lattice on the outermost surface and manually cut surface. (c) Pd 3d highresolution spectrum of PMETAPd-lattice surface. (d) $\mathrm{Cl} 2 \mathrm{p}$ highresolution spectra of PMETAC-lattice surface before and after ion exchange for loading $\mathrm{PdCl}_{4}{ }^{2-}$ moieties.

minute of etching was evident, and after that the atom \% of $\mathrm{C}$ and $\mathrm{O}$ remained almost constant during the entire etching process. We attribute the difference in the chemical composition between the outermost surface and inner to the etching process. To test this, we manually cut $\sim 50 \mu \mathrm{m}$ off and ran the XPS survey scan again. As shown in Figure 1b, the atom $\%$ of $\mathrm{C}, \mathrm{O}$, and $\mathrm{Br}$ is $75.2,23.9$, and $0.2 \%$, respectively, all of which are almost the same as the outermost surface. Also, the high-resolution $\mathrm{Br} 3 \mathrm{~d}$ scan is almost the same between the surface and below $50 \mu \mathrm{m}$. In short, the XPS measurement strongly affirms that $\mathrm{Br}$-initiator-integrated lattices were printed successfully.

The $\mathrm{Br}$ atoms on the surface of Br-lattice then act as initiators to carry out surface-initiated ATRP to grow PMETAC brushes. As shown in Figure 1a, the $\mathrm{Cl} 2 \mathrm{p}$ signal present at $197.1 \mathrm{eV}$ and the sharp increase of $\mathrm{N}$ from 1.0 to $6.5 \%$ is attributed to the $\mathrm{Cl}$ and $\mathrm{N}$ in PMETAC, which indicates the formation of a PMETAC coating on the microattice surface. In addition, the atom $\%$ of $\mathrm{P}$ decreased from $4.0 \%$ to almost zero, suggesting that a dense PMETAC surface coating was formed. All evidence indicates that a uniform and dense PMETAC coating was grafted on the lattice by the surface-initiated ATRP. The grafted PMETAC nanocoating was then used as a platform to immobilize $\mathrm{PdCl}_{4}{ }^{2-}$ moieties by ion exchange because of their high affinity to quaternary ammonium of PMETAC. ${ }^{36}$ The successful loading was confirmed by $\mathrm{Pd} 3 \mathrm{~d}_{5 / 2}$ and $\mathrm{Pd} 3 \mathrm{~d}_{3 / 2}$ signals at 342.1 and $336.7 \mathrm{eV}$, respectively (Figure 1c). Moreover, the increased $\mathrm{Cl}$ concentration from 1.6 to $5.1 \%$ after ion exchange and the significant change of the $\mathrm{Cl} 2 \mathrm{p}$ highresolution scan (Figure 1d) also support the loading of Pd. The loaded palladium moieties act as effective catalytical sites for subsequent ELP of $\mathrm{Cu}$ on lattices. ${ }^{12}$ The appearance of $\mathrm{Cu}$ signals at 932.5, 123.0, and $74.5 \mathrm{eV}$ and the complete disappearance of $\mathrm{N}, \mathrm{Cl}$, and $\mathrm{Pd}$ signals indicate the formation of a dense $\mathrm{Cu}$ layer after ELP (Figure S2). 

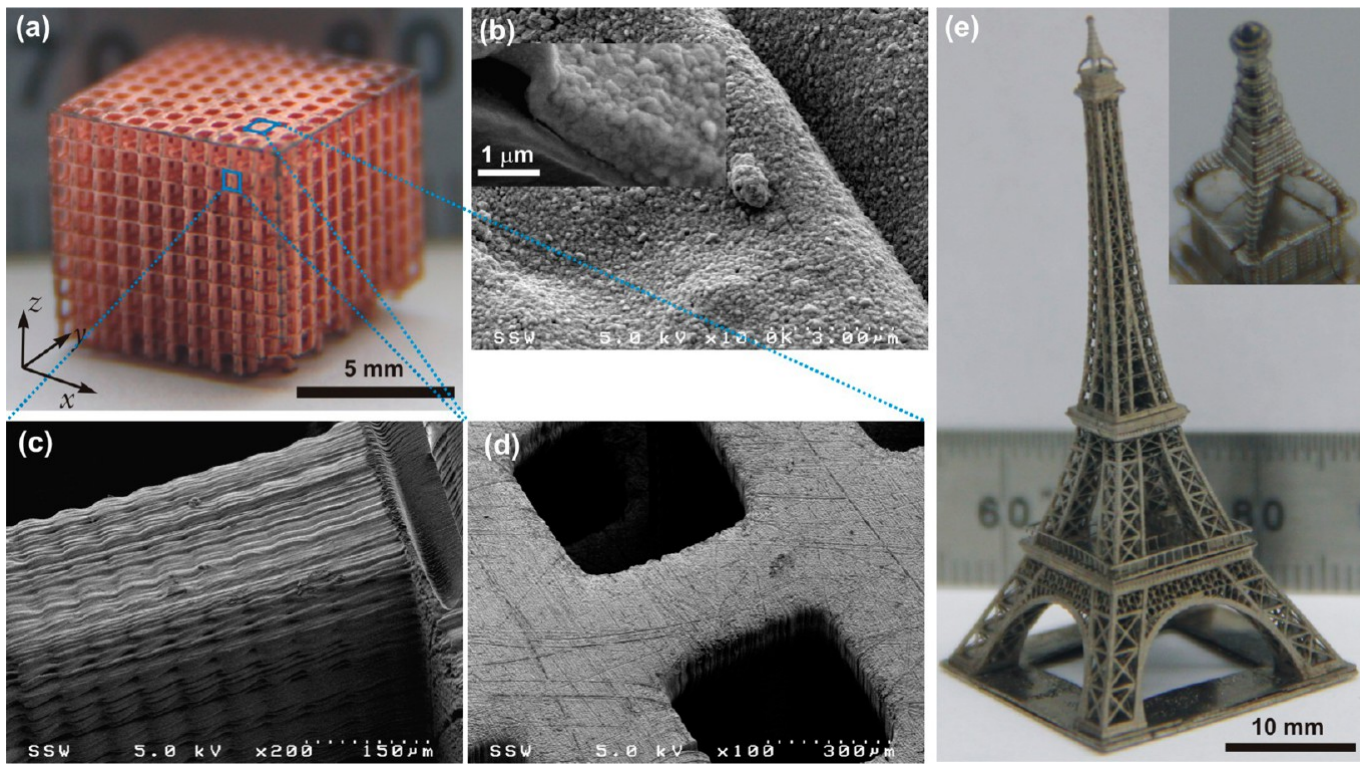

Figure 2. Cu-coated microlattice (a) and its surface morphology in the $x, y$, and $z$ directions $(\mathrm{b}-\mathrm{d})$. (e) Ni-coated Eiffel tower. The inset shows the top of the tower.

Figure 2a shows the resultant Cu-coated shining $8 \times 8 \times 8$ $\mathrm{mm}^{3}$ microlattice. SEM was conducted to investigate the surface morphology of the $\mathrm{Cu}$ layer. It was found that the lattice was coated with a layer of $\mathrm{Cu}$ (Figure $2 \mathrm{~b}-\mathrm{d}$ ) after $25 \mathrm{~min}$ of ELP. The $370 \mathrm{~nm}$ thick layer was composed of dense particles of ca. 100-200 $\mathrm{nm}$ in diameter that uniformly covered the entire lattice surface (Figure 2b). In the $z$ direction (Figure 2c), one can find that the lattice was built layer-by-layer, with each layer having a thickness of $\sim 52 \mu \mathrm{m}$, which is consistent with our set point $(50 \mu \mathrm{m})$ for printing. Importantly, the connections between layers are excellent, without any cracks or delamination, which produced a robust microlattice. It is evident that, compared with the flat surface in the $x$ and $y$ directions (Figure $2 \mathrm{~d}$ ), the column in the $z$ direction is a bit rougher. Typically, for this polyelectrolyte-grafted surface, the obtained metal film is highly dependent on the ELP time. ${ }^{32,33}$ As observed by the SEM images (Figure S3), 15 and 20 min of ELP both produced good $\mathrm{Cu}$-coated lattices with uniform coverage, and the thickness of $\mathrm{Cu}$ layer was ca. 140 and 230 $\mathrm{nm}$, respectively, whereas the $\mathrm{Cu}$ layer obtained at $10 \mathrm{~min}$ of ELP was not very dense, with a thickness of ca. $100 \mathrm{~nm}$. Obvious defects can be found at $10 \mathrm{~min}$ of ELP. This indicates that at least 15 min of ELP is necessary to produce good $\mathrm{Cu}$ coated architectures. Notably, with the computer-aided process, we were able to fabricate various desired metallic objects, for example, Ni-coated hollow balls (Figure S4) and Eiffel towers (Figures 2e and S5). Like the $\mathrm{Cu}$ film, the Ni layer is composed of nanoparticles with diameters of ca. 100-200 nm (Figure S6), which uniformly covered the whole polymeric architechture. To the best of our knowledge, metallic Eiffel towers with not only the shape but also the details were realized for the first time using a $3 \mathrm{D}$ printing approach.

Remarkably, the polymer core of the metallic architectures can be etched away to obtain ultralight cellular metal architectures. As a proof of concept, a $4 \times 4 \times 8 \mathrm{~mm}^{3} \mathrm{Cu}$ coated microlattice was immersed into a $3 \mathrm{M} \mathrm{NaOH}$ aqueous solution. After $24 \mathrm{~h}$ at $60{ }^{\circ} \mathrm{C}$, the $45 \mathrm{mg} \mathrm{Cu}$-coated lattice was reduce to only $1.0 \mathrm{mg}$. An ultralight $\mathrm{Cu}$ lattice with a density of $7.2 \mathrm{mg} / \mathrm{cm}^{3}$ was obtained (Figure S7). Importantly, the microlattice retained its shape very well. As shown in Figure $3 \mathrm{a}-\mathrm{c}$, the nano-thick $\mathrm{Cu}$ layer did not warp or collapse. It is
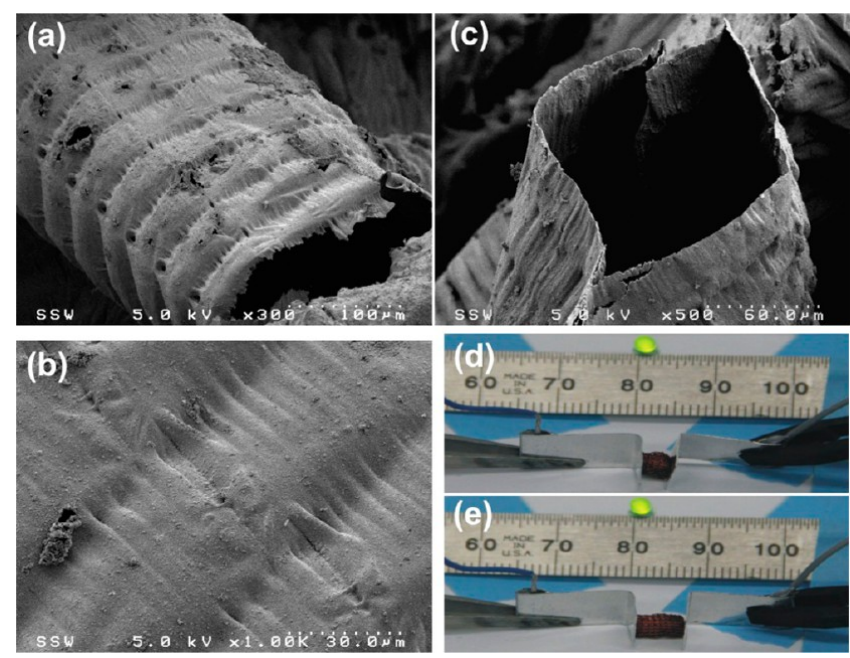

Figure 3. SEM images of $\mathrm{Ni}$ ultralight microlattice obtained by template etching of Ni-coated lattice $(a-c)$ and digital images of a LED-integrated circuit using the Ni ultralight microlattice as an electric wire operated under $50 \%$ compression (d) and after full recovery (e).

well-known that this kind of ultralight metal foam has many unique mechanical, thermal, and energy-absorbing properties such as recovery from deformation..$^{25,26,37,38}$ In our case, to demonstrate the deformation recoverability of the ultralight metallic architecture, we simply integrated the $\mathrm{Cu}$ foam into a circuit with a $9 \mathrm{~V}$ battery and an electric wire to illuminate a commercial LED light. As shown in Figure 3d,e, the LED light remained illuminated even when the foam was compressed to $50 \%$ from full length. When the pressure from the two electrodes was removed gradually, the LED again remained fully illuminated until the foam was fully recovered with zero pressure. More importantly, the foam structure recovered to the orignal shape immediately after the pressure was released, just like a spring. Furthermore, the illuminated LED showed no 
changes even after more than 50 cycles of compressionrelease. This indicates that the $\mathrm{Cu}$ foam has an outstanding ability to fully recover from deformation immediately after the removal of pressure and even after many cycles.

\section{CONCLUSIONS}

We have demonstrated an initiator-integrated 3D printing approach to fabricate metallic architectures with complex geometries. By directly mixing a Br-containing vinyl-terminated initiator into photo-curable resin, 3D printed metallic structures, such as $\mathrm{Cu}$ - and Ni-coated microlattices, hollow balls, and Eiffel towers, were achieved via the surface-initiated ATRP of the printed objects with the initiator integrated and the subsequent polyelectrolyte-brush-assisted ELP of metals. More importantly, our method was also capable of fabricating ultralight cellular metals with desired structures by simply etching the polymer template away. Combining the merits of 3D printing for structure design with those of ATRP for surface modification and polymer-assisted ELP of metals, this universal, robust, and cost-effective approach has greatly extended the capability of 3D printing and will make 3D printing technology more practical in areas of electronics, acoustic absorption, thermal insulation, catalyst supports, and others.

\section{ASSOCIATED CONTENT}

\section{(S Supporting Information}

XPS spectra and SEM images of complex metallic architectures. This material is available free of charge via the Internet at http://pubs.acs.org.

\section{AUTHOR INFORMATION}

\section{Corresponding Author}

*E-mail: jyang@eng.uwo.ca.

\section{Author Contributions}

"These authors contributed equally to this work.

\section{Notes}

The authors declare no competing financial interest.

\section{ACKNOWLEDGMENTS}

We are grateful for the financial support from the Natural Science and Engineering Research Council of Canada (NSERC), the Canada Foundation for Innovation (CFI), the Research Accelerator Grant Program of the University of Western Ontario, the National Natural Science Foundation of China (51171202 and 21125316), and the oversea project of Chinese Academy of Sciences.

\section{REFERENCES}

(1) Geissler, M.; Xia, Y. N. Adv. Mater. 2004, 16, 1249-1269.

(2) Villar, G.; Graham, A. D.; Bayley, H. Science 2013, 340, 48-52.

(3) Derby, B. Science 2012, 338, 921-926.

(4) Duarte Campos, D. F.; Blaeser, A.; Weber, M.; Jakel, J.; Neuss, S.; Jahnen-Dechent, W.; Fischer, H. Biofabrication 2013, 5, 015003.

(5) Ovsianikov, A.; Gruene, M.; Pflaum, M.; Koch, L.; Maiorana, F.; Wilhelmi, M.; Haverich, A.; Chichkov, B. Biofabrication 2010, 2, 014104.

(6) Ahn, S. H.; Lee, H. J.; Kim, G. H. Biomacromolecules 2011, 12, 4256-4263.

(7) Fedorovich, N. E.; Swennen, I.; Girones, J.; Moroni, L.; van Blitterswijk, C. A.; Schacht, E.; Alblas, J.; Dhert, W. J. A. Biomacromolecules 2009, 10, 1689-1696.

(8) Wu, C.; Fan, W.; Zhou, Y.; Luo, Y.; Gelinsky, M.; Chang, J.; Xiao, Y. J. Mater. Chem. 2012, 22, 12288-12295.
(9) Woesz, A.; Rumpler, M.; Stampfl, J.; Varga, F.; Fratzl-Zelman, N.; Roschger, P.; Klaushofer, K.; Fratzl, P. Mater. Sci. Eng., C 2005, 25, 181-186.

(10) Stampfl, J.; Liska, R. Macromol. Chem. Phys. 2005, 206, 12531256.

(11) Kitson, P. J.; Rosnes, M. H.; Sans, V.; Dragone, V.; Cronin, L. Lab Chip 2012, 12, 3267-3271.

(12) Sun, K.; Wei, T. S.; Ahn, B. Y.; Seo, J. Y.; Dillon, S. J.; Lewis, J. A. Adv. Mater. 2013, 25, 4539-4543.

(13) Lopes, A. J.; MacDonald, E.; Wicker, R. B. Rapid Prototyping J. 2012, 18, 129-143.

(14) Derby, B. Annu. Rev. Mater. Res. 2010, 40, 395-414.

(15) Sirringhaus, H.; Shimoda, T. MRS Bull. 2003, 28, 802-803.

(16) Pham, D. T.; Gault, R. S. Int. J. Mach. Tools Manuf. 1998, 38, 1257-1287.

(17) Kitson, P. J.; Symes, M. D.; Dragone, V.; Cronin, L. Chem. Sci. 2013, 4, 3099-3103.

(18) Symes, M. D.; Kitson, P. J.; Yan, J.; Richmond, C. J.; Cooper, G. J. T.; Bowman, R. W.; Vilbrandt, T.; Cronin, L. Nat. Chem. 2012, 4, 349-354.

(19) Wang, X.; Cai, X.; Guo, Q.; Zhang, T.; Kobe, B.; Yang, J. Chem. Commun. 2013, 49, 10064-10066.

(20) Guo, Q.; Cai, X.; Wang, X.; Yang, J. J. Mater. Chem. B 2013, 1, 6644-6649.

(21) Ko, S. H.; Chung, J.; Hotz, N.; Nam, K. H.; Grigoropoulos, C. P. J. Micromech. Microeng. 2010, 20, 125010.

(22) Kim, M. S.; Chu, W. S.; Kim, Y. M.; Avila, A. P. G.; Ahn, S. H. Int. J. Precis. Eng. Manuf. 2009, 10, 147-150.

(23) Sanchez-Romaguera, V.; Madec, M. B.; Yeates, S. G. React. Funct. Polym. 2008, 68, 1052-1058.

(24) Wang, J.; Auyeung, R. C.; Kim, H.; Charipar, N. A.; Pique, A. Adv. Mater. 2010, 22, 4462-4466.

(25) Schaedler, T. A.; Jacobsen, A. J.; Torrents, A.; Sorensen, A. E.; Lian, J.; Greer, J. R.; Valdevit, L.; Carter, W. B. Science 2011, 334, 962-965.

(26) Torrents, A.; Schaedler, T. A.; Jacobsen, A. J.; Carter, W. B.; Valdevit, L. Acta Mater. 2012, 60, 3511-3523.

(27) Lian, J.; Jang, D.; Valdevit, L.; Schaedler, T. A.; Jacobsen, A. J.; Carter, W. B.; Greer, J. R. Nano Lett. 2011, 11, 4118-4125.

(28) Liu, X.; Wu, Y.; Gao, Y.; Wang, J.; Li, Z.; Han, J.; Jin, G.; Ma, H. J. Mater. Chem. 2012, 22, 6327-6334.

(29) Li, Z.; Zhang, S.; Zhang, P.; Yang, D.; Jin, G.; Ma, H. Polym. Adv. Technol. 2012, 23, 1240-1245.

(30) Wu, Y.; Huang, Y.; Ma, H. J. Am. Chem. Soc. 2007, 129, 72267227.

(31) Azzaroni, O.; Fonticelli, M. H.; Benitez, G.; Schilardi, P. L.; Gago, R; Caretti, I.; Vazquez, L.; Salvarezza, R. C. Adv. Mater. 2004, $16,405-409$.

(32) Wang, X.; Hu, H.; Shen, Y.; Zhou, X.; Zheng, Z. Adv. Mater. 2011, 23, 3090-3094.

(33) Wang, X.; Zhang, T.; Kobe, B.; Lau, W. M.; Yang, J. Chem. Commun. 2013, 49, 4658-4660.

(34) Liu, X. Q.; Zhou, X. C.; Li, Y.; Zheng, Z. J. Chem.-Asian J. 2012, 7, 862-870.

(35) Guo, R.; Yu, Y.; Xie, Z.; Liu, X.; Zhou, X.; Gao, Y.; Liu, Z.; Zhou, F.; Yang, Y.; Zheng, Z. Adv. Mater. 2013, 25, 3343-3350.

(36) Ye, Q.; Wang, X. L.; Hu, H. Y.; Wang, D. A.; Li, S. B.; Zhou, F. J. Phys. Chem. C 2009, 113, 7677-7683.

(37) Sun, H.; Xu, Z.; Gao, C. Adv. Mater. 2013, 25, 2554-2560.

(38) Tappan, B. C.; Huynh, M. H.; Hiskey, M. A.; Chavez, D. E.; Luther, E. P.; Mang, J. T.; Son, S. F. J. Am. Chem. Soc. 2006, 128, 6589-6594. 